\title{
Estradiol attenuates the TGF- $\beta 1$-induced conversion of primary TAFs into myofibroblasts and inhibits collagen production and myofibroblast contraction by modulating the Smad and Rho/ROCK signaling pathways
}

\author{
HE-SONG JIANG $^{1}$, LEI-LEI ZHU ${ }^{1}$, ZHENG ZHANG $^{1}$, HAI CHEN $^{2}$, YUN CHEN ${ }^{2}$ and YU-TIAN DAI ${ }^{2}$ \\ ${ }^{1}$ Medical School, Nanjing University; ${ }^{2}$ Department of Andrology, Nanjing Drum Tower Hospital, \\ Nanjing Medical University, Nanjing, Jiangsu, P.R. China
}

Received March 23, 2015; Accepted July 6, 2015

DOI: $10.3892 /$ ijmm.2015.2288

\begin{abstract}
The transformation of tunica albuginea-derived fibroblasts (TAFs) into myofibroblasts plays an important role in the pathological progress of Peyronie's disease (PD). However, no treatment which addresses this transformation is currently available. Estrogen has been shown to inhibit the progression of fibrosis in a number of fibrotic diseases. The aim of this study was to determine whether estrogen [17 $\beta$-estradiol (E2)] suppresses the diffentiation of primary rat TAFs into myofibroblasts in vitro. TAFs obtained from male Sprague-Dawley rats were stimulated with either transforming growth factor- $\beta 1$ (TGF- $\beta 1$ ) or E2. Western blot analysis and immunofluorescence staining were used to assess changes in the expression levels of $\alpha$-smooth muscle actin ( $\alpha$ SMA). The expression levels of additional proteins (GAPDH, p-Smad2, Smad2, Smad4, RhoA, Rac1, ROCK1 and ROCK2) were also measured by western blot analysis. We used collagen gel assays to assess cell contractility. Additionally, the concentration of hydroxyproline in the TAF cell culture medium was detected using commercially available kits. We found that E2 reduced $\alpha$ SMA expression which was induced by TGF- $\beta 1$. E2 also suppressed the TGF- $\beta 1$-induced increase in the concentration of hydroxyproline (a marker of collagen) in addition to suppressing the contraction of TAFs. The key processes affected by TGF- $\beta 1$ treatment included the phosphorylation of Smad2, ras homolog gene family, member A (RhoA) and Rho-associated, coiled-coil
\end{abstract}

Correspondence to: Dr Yu-Tian Dai, Department of Andrology, Nanjing Drum Tower Hospital, Nanjing Medical University, 321 Zhongshan Road, Nanjing, Jiangsu 210008, P.R. China

E-mail: yutiandai705@126.com

Abbreviations: E2, 17 $\beta$-estradiol; TAFs, tunica albuginea-derived fibroblasts; PD, Peyronie's disease; $\alpha$ SMA, $\alpha$-smooth muscle actin; ECM, extracellular matrix; TGF- $\beta 1$, transforming growth factor- $\beta 1$

Key words: estrogen, Peyronie's disease, transforming growth factor- $\beta 1$, myofibroblasts, tunica albuginea-derived fibroblast containing protein kinase 2 (ROCK2); this increase in phosphorylation was inhibited by treatment with E2. Collectively, these results demonstrate that by modulating the activation of the TGF- $31-$ Smad and RhoA-ROCK2 signaling pathways, E2 inhibited the transformation of TAFs into myofibroblasts, decreased the expression of collagen and suppressed the contraction of myofibroblasts in response to TGF- $\beta 1$ stimulation.

\section{Introduction}

Peyronie's disease (PD) is a localized fibrotic condition of the tunica albuginea (TA) that is often characterized by the presence of a fibrous plaque containing an excessive amount of collagen, and by fibroblast proliferation, which leads to penile deformity and curvature, as well as potential erectile dysfunction (1). It has been hypothesized that PD arises as a result of a disordered wound-healing response to repetitive micro-injuries of the TA (2).

Previous studies involving patients with PD have demonstrated that the transformation of TA-derived fibroblasts (TAFs) into $\alpha$-smooth muscle actin ( $\alpha$ SMA)-positive myofibroblasts is key to the development of fibrous plaques and that this process is promoted by the accumulation of transforming growth factor- $\beta 1$ (TGF- $\beta 1)(3,4)$. During the wound-healing process, myofibroblasts secrete extracellular matrix (ECM) components (particularly collagen) and contract by using smooth muscle actin-myosin complexes to speed up wound repair. These cells undergo apoptosis after the healing process is complete (5). However, the persistence of myofibroblasts leads to ECM expansion and enhanced contractility, which is observed in several fibrotic diseases. In PD, the excessive secretion of ECM components and the concurrent contraction of myofibroblasts result in the respective development of fibrous plaques and penile curvature $(6,7)$. The Smad-dependent and Smad-independent signaling pathways are both involved in the activation of myofibroblasts, which occurs through the interaction between TGF- $\beta 1$ and its receptor (TGF- $\beta 1 \mathrm{R})$ (8). Activated TGF- $\beta 1 \mathrm{R}$ then phosphorylates Smad2 and Smad3, which in turn form a complex with Smad4. These activated Smad complexes translocate to the nucleus where they regulate the 
transcription of target genes and accelerate the production of collagen (9). Additionally, the Smad-independent ras homolog gene family, member A (RhoA)/Rho-associated, coiled-coil containing protein kinase (ROCK) pathway is a primary regulator of myofibroblast shape, polarity and contraction through the modulation of actin polymerization, actomyosin contractility, cell adhesion and microtubule dynamics (10). Therefore, preventing the transformation of fibroblasts into myofibroblasts may be a useful strategy for suppressing the TA fibrotic-remodeling events that ultimately lead to PD.

Previous studies have demonstrated that the development of human fibrotic diseases differs according to gender: women are generally more resistant to fibrosis than men (11). For example, Yang et al demonstrated that pre-menopausal women have a lower risk of developing severe liver fibrosis compared to men and post-menopausal women (12). An increasing body of evidence has demonstrated that estrogen inhibits the activation of fibroblasts and fibrogenesis in a number of organs, including the liver (13), heart (14), lungs (15) and kidneys (16). However, the specific details of how estrogen effects the fibrotic process or fibroblast transformation remain controversial. Novotny et al found that estrogen receptor (ER) agonists induced the transformation of fibroblasts into myofibroblasts and increased the production of ECM components in ovariectomized rats (17). Therefore, the effects of estrogen on the fibrotic disease process may be related to the type of organ or tissue affected.

To the best of our knowledge, no research studies have been conducted to date to investigate the mechanisms through which estrogen affects the pathological and physiological process of PD. Therefore, the aim of the present study was to determine the specific effects of estrogen [17 $\beta$-estradiol (E2)] on the functions of cultured TAFs and to explore the mechanisms of action of TGF- $\beta 1$ in TAFs in vitro. Additionally, we sought to determine whether E2 regulates the secretion of collagen through the TGF- $\beta$-Smad signaling pathway and/or the contraction of myofibroblasts through the RhoA-ROCK signaling pathway.

\section{Materials and methods}

Cell culture. In independent experiments, 3 male SpragueDawley rats (from the Animal Feeding Center of Nanjing Medical University, Jiangsu, China) were used to isolate the TAFs. Under $4 \%$ chloral hydrate anesthesia, penile tissue was harvested to isolate the TAFs and then all rats were euthanized. Primary TAFs were isolated, cultured and identified as previously described (18); cells at passage 8 or less were used in the experiments. Briefly, TAFs from Sprague-Dawley rats (Animal Feeding Center of Nanjing Medical University, Jiangsu, China) were cultured in low-glucose Dulbecco's modified Eagle's medium (DMEM), supplemented with $10 \%$ fetal bovine serum (FBS; both from Gibco, Carlsbad, CA, USA) and 1\% antibiotics $\left(100 \mathrm{mg} / \mathrm{l}\right.$ streptomycin and $100 \mathrm{U} / \mathrm{ml}$ penicillin) at $37^{\circ} \mathrm{C}$ in a humidified $95 \%$ air $/ 5 \% \mathrm{CO}_{2}$ environment. When the TAFs reached $80 \%$ confluence, they were routinely digested by $0.25 \%$ trypsin and then passaged at a 1:2 dilution. All procedures were approved by the Institutional Animal Care and Use Committee of Nanjing University. The TAFs were then cultured in serum-free medium and treated with E2 (Sigma, St. Louis, MO, USA) at a concentration of 1-100 nM. TGF- $\beta 1(5-10 \mathrm{ng} / \mathrm{ml}$; Sigma) was added at the same time as E2. Various concentra- tions of TGF (0, 5 and $10 \mathrm{ng} / \mathrm{ml})$ and E2 (0, 10 and $100 \mathrm{nM})$ were used to explore the dose-dependent response. Finally, we found that $5 \mathrm{ng} / \mathrm{ml}$ of TGF induced the conversion of TAFs into myofibroblasts and that $10 \mathrm{nM}$ of E2 effectively attenuated this process in our repeated independent experiments. Moreover, this dose is consistent with that used in previous research (19-21). The control cells were exposed to the vehicle (DMEM; referred to untreated or normal control). The cells were then grown for either $24 \mathrm{~h}$ or for corresponding amounts of time.

Collagen gel contraction assay. Collagen gels were prepared using $2 \mathrm{mg} / \mathrm{ml}$ of rat tail collagen I (Wobio, Nanjing, China) that was neutralized with $1 \mathrm{M} \mathrm{NaOH}$ and supplemented with DMEM. Gels contained $2-3 \mathrm{mg} / \mathrm{ml}$ of rat tail collagen in $0.5-\mathrm{ml}$ aliquots; the amount of collagen used depended upon the experimental protocol employed. The TAFs were seeded at a density of $3 \times 10^{5}$ cells $/ \mathrm{ml}$ in microtiter plates that were lubricated with FBS. Following lubrication, $0.5 \mathrm{ml}$ of the final collagen gel was incubated at $37^{\circ} \mathrm{C}$ in a humidified $95 \%$ air $/ 5 \% \mathrm{CO}_{2}$ environment for $24 \mathrm{~h}$. Images were acquired using an Odyssey Scanning System (LI-COR Biosciences, Lincoln, NE, USA), and the surface areas were quantified using ImageJ software (NIH, Bethesda, MD, USA).

Measurement of hydroxyproline concentration. Hydroxyproline was used to measure the total collagen secreted into the medium according to the method described in the study by Woessner (22) and the protocol included in the hydroxyproline kit (A030-1; Nanjing Jiancheng Bioengineering Institute, Jiangsu, China). Duplicate 300- $\mu$ l aliquots of medium were taken from each sample and transferred to microtiter plates, and the absorbance of each was determined at $550 \mathrm{~nm}$ by spectrophotometry. The hydroxyproline concentrations of the sample pending to be tested were calculated using a linear standard curve and are presented as $\mu \mathrm{g} / \mathrm{ml}$ cell culture medium.

Western blot analysis. Cellular proteins were obtained from the TAFs. Western blot analysis of these proteins was performed as previously described (23). Briefly, the TAFs were washed twice with phosphate-buffered saline (PBS) and then lysed in RIPA buffer (Sigma). Total protein concentrations were measured using bicinchoninic acid (BCA) reagent (Beyotime Biotech, Jiangsu, China). Proteins were separated by $10 \%$ sodium dodecyl sulfate-polyacrylamide gel electrophoresis (SDS-PAGE) and were then electrophoretically transferred onto polyvinylidene fluoride (PVDF) membranes. The membranes were incubated at $37^{\circ} \mathrm{C}$ for $1 \mathrm{~h}$ in blocking solution containing $5 \%$ non-fat milk in TBST. The membranes were then incubated at $4^{\circ} \mathrm{C}$ overnight with the relevant primary antibodies [anti- $\alpha$ SMA (SAB5500002), anti-Smad2 (SAB2103328), anti-phosphorylated (p-)Smad2 (SAB4300251), anti-Smad4 (SAB4200405), anti-RhoA (WH0000387M3), anti-ras-related $\mathrm{C} 3$ botulinum toxin substrate 1 (Rac1; SAB4300461), anti-ROCK1 (R6028), anti-ROCK2 (R8653) and anti-GAPDH (G9295); all from Sigma] at a dilution of 1:1,000. After washing the membranes with TBST, they were then incubated with the secondary antibody (horseradish peroxidase conjugated goat anti-rabbit/mouse IgG; Wuhan Boster Biological Technology Ltd., Wuhan, China) at room temperature for $2 \mathrm{~h}$. The band densities were detected using enhanced 
chemiluminescence (ECL) reagents (Beyotime Biotech) and an Odyssey Scanning System (LI-COR Biosciences). We then used ImageJ software to quantify the expression levels of the target proteins by calculating the ratio of the mean intensity of each target protein relative to that of GAPDH.

Immunofluorescence staining. Immunofluorescence staining for $\alpha$ SMA was performed using the cultured TAFs as previously described (23). Briefly, the TAFs on the coverslips were fixed with $4 \%$ paraformaldehyde, washed 3 times with pre-chilled PBS and permeabilized with $3 \%$ bovine serum albumin (BSA) in PBS containing $0.3 \%$ Triton X-100 for $30 \mathrm{~min}$ at room temperature. Following this, the TAFs were incubated with primary antibody (anti- $\alpha$ SMA) overnight at $4^{\circ} \mathrm{C}$. After being washed 3 times with PBS, the TAFs were incubated with $0.1 \%$ DAPI and secondary Rhodamine Red-X labeled antibody (Invitrogen/Life Technologies, Carlsbad, CA, USA) at a dilution of 1:500 for $60 \mathrm{~min}$ at room temperature, after which they were again washed with PBS. The coverslips were then transferred onto glass slides and imaged using a fluorescence microscope (Olympus, Tokyo, Japan).

Statistical analysis. All data were obtained from at least 3 individual experiments. The experimental results are expressed as the mean values \pm standard deviation (SD). Statistical analysis was performed by one-way analysis of variance (ANOVA) using Microsoft Excel 2010 and GraphPad software. A P-value $<0.05$ was considered to indicate a statistically significant difference.

\section{Results}

TGF- $\beta 1$ promotes the transformation of TAFs into myofibroblasts. In independent experiments with TAFs obtained from 3 different Sprague-Dawley rats, TGF- $\beta 1$ significantly increased the expression of $\alpha$ SMA (a myofibroblast marker) in a concen-

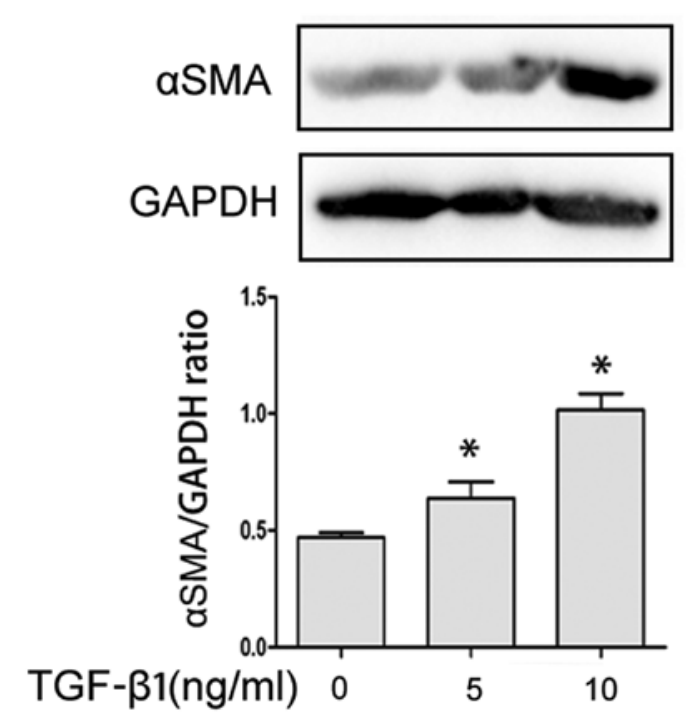

Figure 1. Effects of transforming growth factor- $\beta 1$ (TGF- $\beta 1$ ) on the expression of $\alpha$-smooth muscle actin ( $\alpha$ SMA) in tunica albuginea-derived fibroblasts (TAFs). Representative western blots showing the total protein levels of $\alpha$ SMA in response to the dose-dependent application of TGF- $\beta 1$ to TAFs. The relative levels of $\alpha$ SMA to GAPDH are indicated by the corresponding bar chart. ${ }^{*} \mathrm{P}<0.01$ vs. untreated controls. Results are based on data from 3 independent experiments.

tration-dependent manner compared to the TAFs which were not treated with TGF- $\beta 1$ and exposed to the vehicle (1\% DMEM). The above results were obtained by western blot analysis (Fig. 1).

Supplementation of E2 attenuates the TGF- $\beta 1$-induced expression of $\alpha S M A$ in TAFs. Since the expression of $\alpha \mathrm{SMA}$ is a hallmark indicator that can be used to identify myofibroblasts, the effects of $\mathrm{E} 2$ on TGF- $\beta 1$-induced $\alpha$ SMA protein levels
A

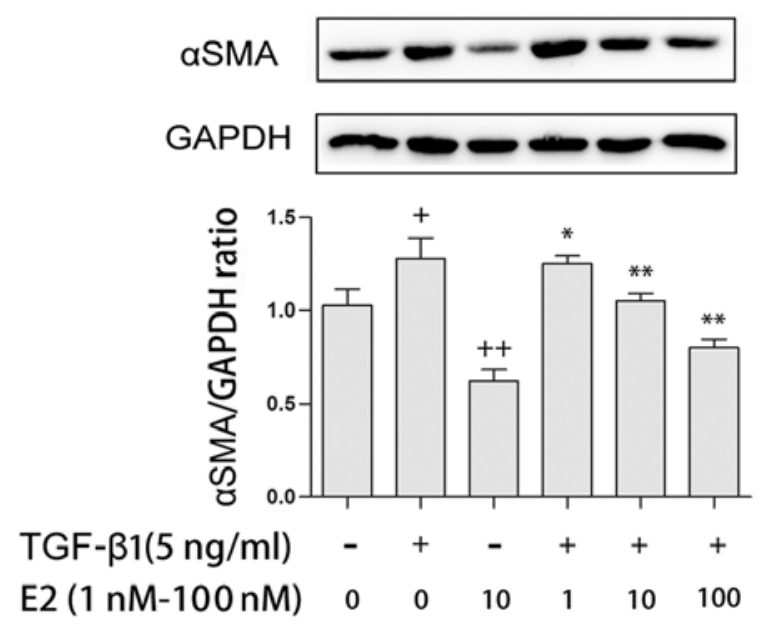

B
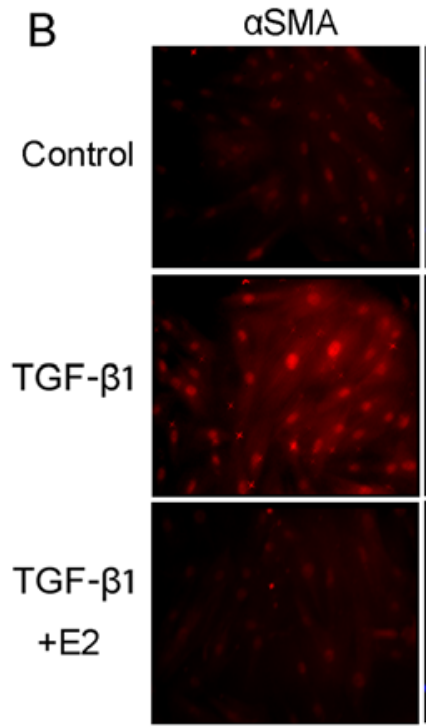

DAPI
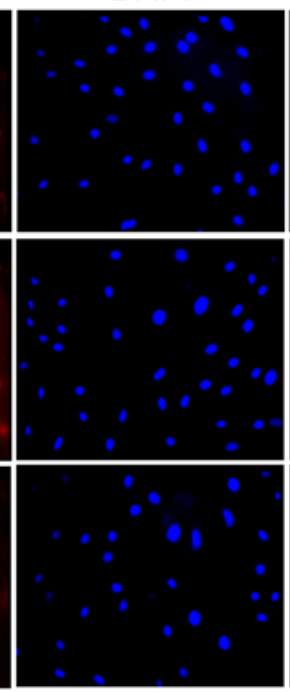

Merge

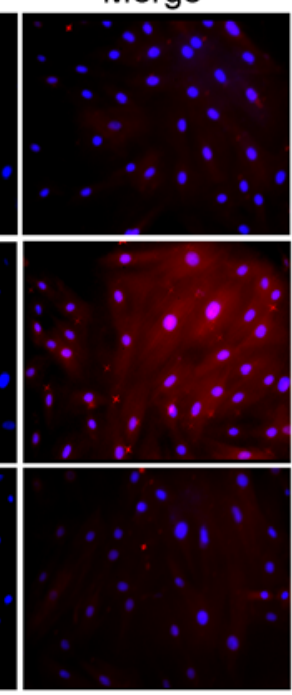

Figure 2. Treatment of tunica albuginea-derived fibroblasts (TAFs) with $17 \beta$-estradiol (E2) attenuates transforming growth factor- $\beta 1$ (TGF- $\beta 1$ )-induced $\alpha$-smooth muscle actin ( $\alpha$ SMA) expression. (A) Representative western blots showing the phosphorylation and total protein levels of $\alpha$ SMA in TAFs treated with TGF- $\beta 1$ alone or TAFs treated with TGF- $\beta 1$ and E2. ${ }^{*} \mathrm{P}<0.01$ and ${ }^{* *} \mathrm{P}<0.001$ vs. TGF- $\beta 1$ stimulated cells; ${ }^{++} \mathrm{P}<0.001$ vs. untreated controls. Quantitative data are presented as the means \pm SD. Three independent experiments were performed. (B) Representative images of the immunofluorescence staining of $\alpha$ SMA in TAFs incubated with TGF- $\beta 1(5 \mathrm{ng} / \mathrm{ml})$ alone or with TGF- $\beta 1(5 \mathrm{ng} / \mathrm{ml})$ and E2 $(10 \mathrm{nM})$. Red fluorescence indicates $\alpha$ SMA, and blue fluorescence represents the nuclei of TAFs. 


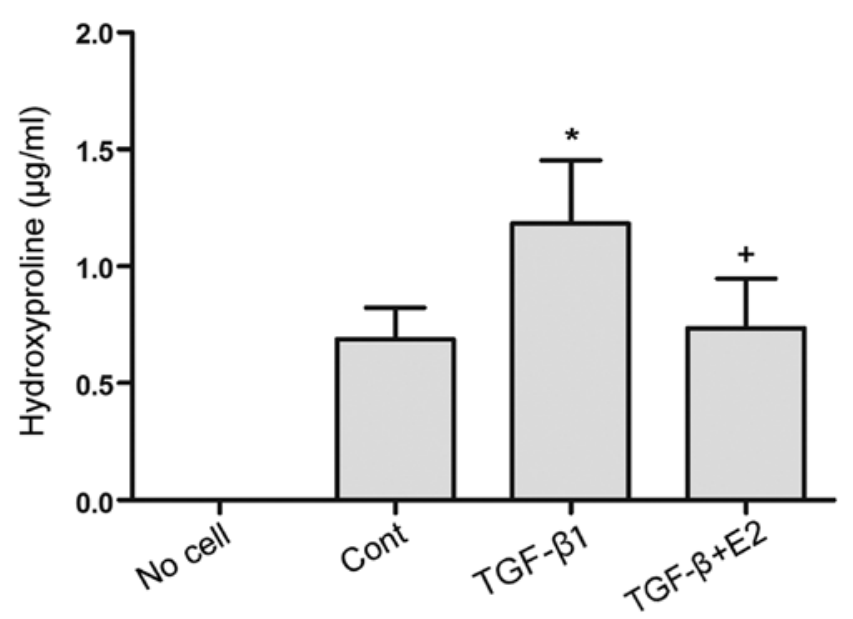

Figure 3. 17 $\beta$-estradiol (E2) attentuates the increase in the concentration of hydroxyproline induced by transforming growth factor- $\beta 1$ (TGF- $\beta 1$ ) in tunica albuginea-derived fibroblasts (TAFs). The total levels of hydroxyproline present in the TAF culture medium were measured using the hydroxyproline assay described in Materials and methods at $24 \mathrm{~h}$ after treatment with TGF- $\beta 1(5 \mathrm{ng} / \mathrm{ml})$ alone or with TGF- $\beta 1(5 \mathrm{ng} / \mathrm{ml})$ and E2 $(10 \mathrm{nM})$. The results are expressed as the means \pm SD and are based on 3 independent experiments. ${ }^{*} \mathrm{P}<0.01$ and ${ }^{*} \mathrm{P}<0.01$ vs. untreated controls (Cont); ${ }^{+} \mathrm{P}<0.01$ vs. TGF- $\beta 1$-stimulated cells.

were measured by western blot analysis and the immunofluorescence staining of TAFs. Treatment with E2 (1-100 nM) reduced the expression of $\alpha \mathrm{SMA}$ in a dose-dependent manner in both the presence and absence of TGF- $\beta 1(5 \mathrm{ng} / \mathrm{ml}$; Fig. 2A). Consistent with these results, treatment with $10 \mathrm{nM}$ E2 reduced $\alpha$ SMA protein expression in both the presence and absence of TGF- $\beta 1$, as determined by immunofluorescence staining (Fig. 2B).

E2 reduces the levels of TGF- $\beta 1$-induced hydroxyproline in $T A F s$. Hydroxyproline is generally considered to be an amino acid specific to collagen proteins, and it can therefore be used to measure fibrosis (22). As shown in Fig. 3, stimulation with TGF- $\beta 1(5 \mathrm{ng} / \mathrm{ml})$ led to elevated levels of hydroxyproline in the TAFs compared to the controls $(\mathrm{P}<0.01)$. We then examined the effects of E2 on the TGF- $\beta 1$-mediated induction of collagen secretion in TAFs and revealed that treatment with E2 $(10 \mathrm{nM})$ suppressed the TGF- $\beta 1$-induced increase in hydroxyproline levels in these cells $(\mathrm{P}<0.01)$.

TGF- $\beta 1$-induced collagen gel contraction is suppressed by $E 2$. We carried out a collagen gel contraction assay to analyze the inhibitory effects of E2 on the contractile process. The TAFs mixed with E2 were applied to collagen gels and incubated with or without TGF- $\beta 1$ for $24 \mathrm{~h}$. The collagen gels were prepared as follows: i) normal control; ii) collagen gel with $5 \mathrm{ng} / \mathrm{ml}$ TGF- $\beta 1$; and iii) collagen gel with $5 \mathrm{ng} / \mathrm{ml}$ TGF- $\beta 1$ and $10 \mathrm{nM}$ E2. TGF- $\beta 1$ significantly enhanced gel contraction compared to the vehicle control $(\mathrm{P}<0.001$; Fig. 4). Although E2 significantly suppressed gel contraction at a concentration of $10 \mathrm{nM}(\mathrm{P}<0.001)$ in the presence of TGF- $\beta 1(5 \mathrm{ng} / \mathrm{ml})$, it did not completely reverse this contraction $(\mathrm{P}<0.01$; Fig. 4).

E2 decreases TGF- $\beta 1$-induced Smad signaling. In exploring the molecular mechanisms underlying the negative role of $\mathrm{E} 2$ in relation to the functioning of TAFs, we discovered that

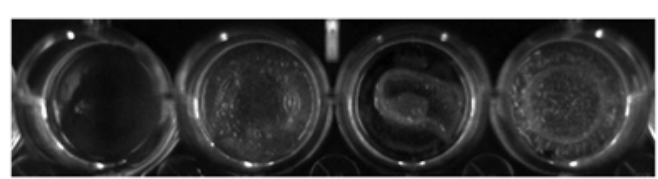

Surface area quantification

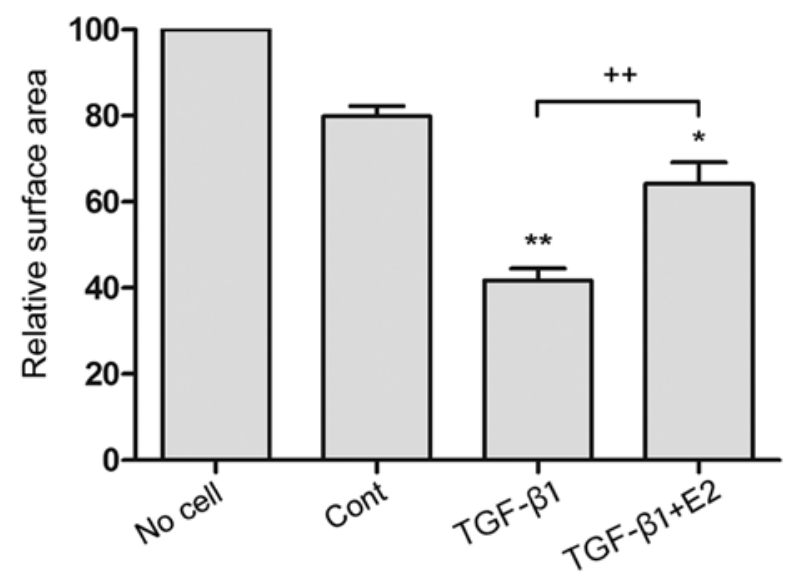

Figure 4. Effects of $17 \beta$-estradiol (E2) on tunica albuginea-derived fibroblast (TAF) contraction following stimulation with transforming growth factor- $\beta 1$ (TGF- $\beta 1$ ). By $24 \mathrm{~h}$, TAFs seeded in $2.5 \mathrm{mg} / \mathrm{ml}$ of rat tail collagen were found to spontaneously contract the collagen gels. A subsequent $24 \mathrm{~h}$-long treatment with TGF- $\beta 1(5 \mathrm{ng} / \mathrm{ml})$ or TGF- $\beta 1(5 \mathrm{ng} / \mathrm{ml})$ plus E2 $(10 \mathrm{nM})$ partly reversed spontaneous collagen gel contraction and also increased collagen gel surface area compared with untreated controls (Cont) by 50 and $80 \%$, respectively. ${ }^{*} \mathrm{P}<0.01$ and ${ }^{* *} \mathrm{P}<0.001$ vs. untreated controls; ${ }^{++} \mathrm{P}<0.001$ vs. TGF- $\beta 1$-stimulated cells. Quantitative data are presented as the means \pm SD. Three independent experiments were performed.

the levels of $\mathrm{p}$-Smad2 were enhanced in the TAFs following stimulation with TGF- $\beta 1(5 \mathrm{ng} / \mathrm{ml})$; this effect was attenuated following incubation with increasing doses of E2 (1-100 nM; Fig. 5A). Additionally, no significant differences in the total protein levels of Smad 2 and Smad4 were noted between the TAFs incubated with TGF- $\beta 1$ or the TAFs treated with E2 (Fig. 5A and B).

The TGF- $\beta 1$-induced activation of the Rho/ROCK pathway is inhibited by E2. The Rho/ROCK signaling pathway promotes cell contraction and migration and is a major effector of fibrotic disease (24-26). We thus performed an in vitro experiment in which the cells were pre-treated with TGF- $\beta 1(5 \mathrm{ng} / \mathrm{ml})$ for $24 \mathrm{~h}$ and then treated with $10 \mathrm{nM}$ E2 for $0,2,6,24,48$ and $72 \mathrm{~h}$. The expression of RhoA decreased after $2 \mathrm{~h}$ and was completely depleted by $24 \mathrm{~h}$ (Fig. 5C). To elucidate the molecular mechanisms responsible for this, we examined the expression of proteins of the Rho/ROCK signaling pathway, which include RhoA, Rac1, ROCK1 and ROCK2 (Fig. 5D). We found that only RhoA was upregulated after $24 \mathrm{~h}$ of stimulation with TGF- $\beta 1(5 \mathrm{ng} / \mathrm{ml})$. It should be noted that the expression levels of RhoA and ROCK were decreased in the TAFs following incubation with E2 in a dose-dependent manner compared to the TAFs which received only TGF- $\beta 1$ and that obvious changes to the total protein levels of Rac1, ROCK1 and ROCK2 were demonstrated (Fig. 5D). These results suggest that E2 suppresses the TGF- $\beta 1$-induced activation of the RhoA/ROCK2 signaling pathway. 


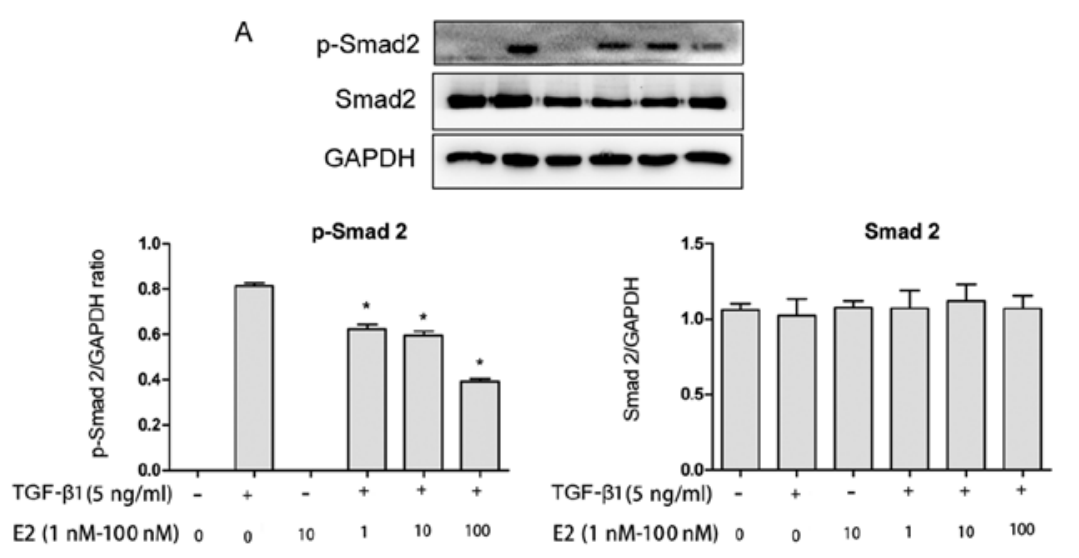

B
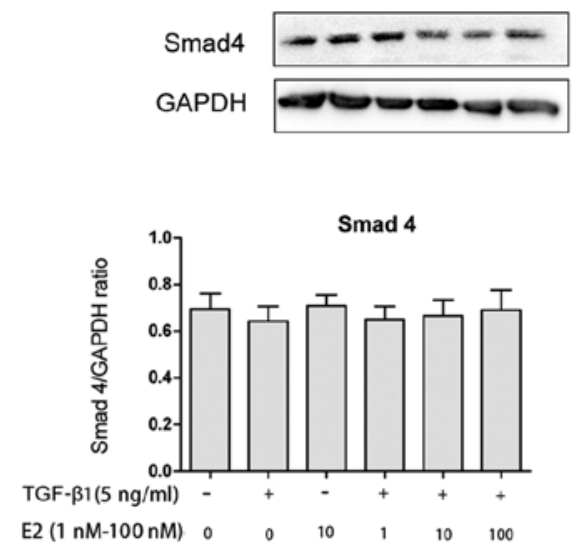

C
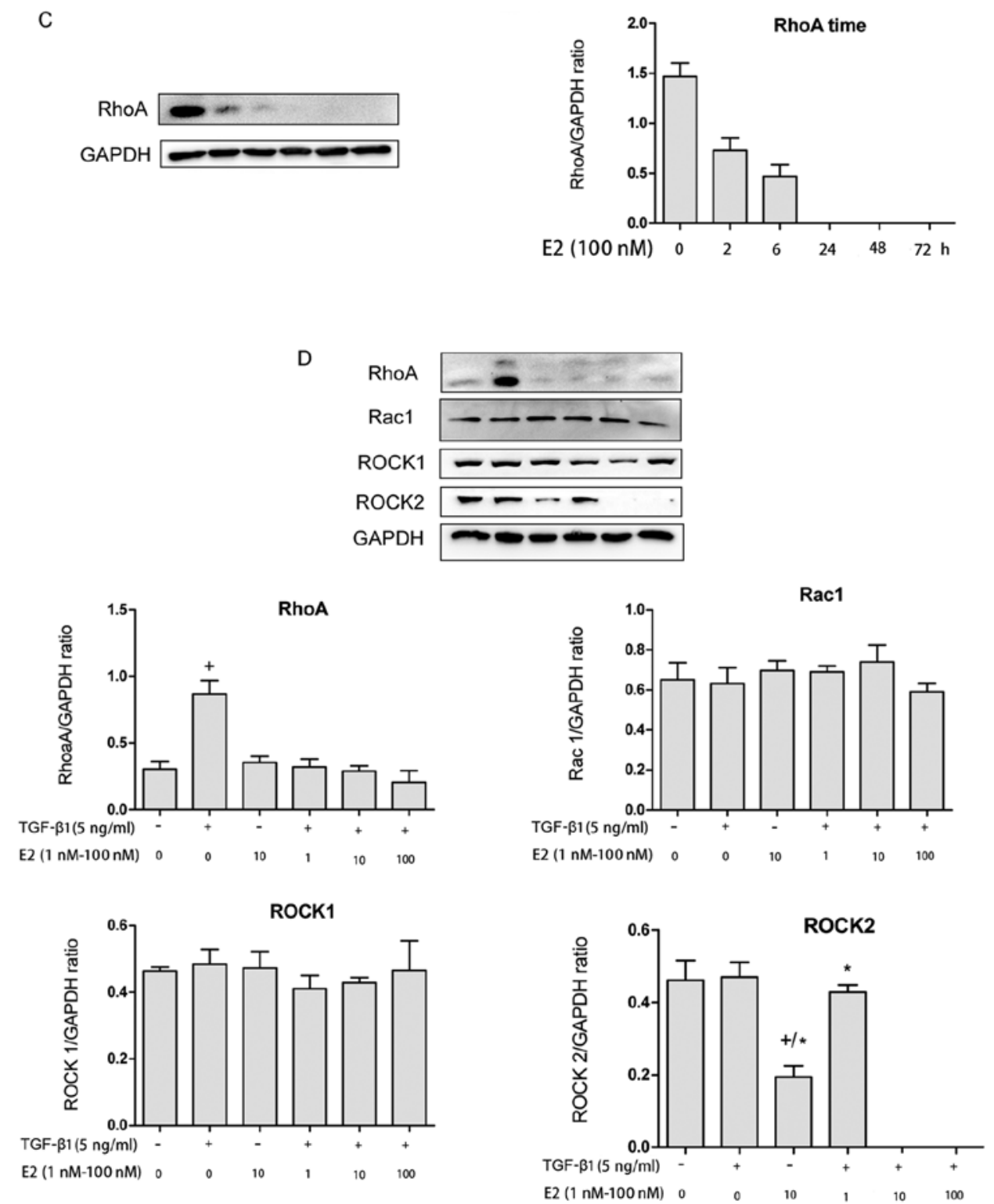

Figure 5. Effects of $17 \beta$-estradiol (E2) on the transforming growth factor- $\beta 1$ (TGF- $\beta 1$ )-induced activation of Smad-dependent and Smad-independent (RhoA)/ROCK) signaling pathways. (A) Representative western blots showing the phosphorylation status and total protein levels of Smad2 in tunica albugineaderived fibroblasts (TAFs) treated with TGF- $\beta 1$ alone or with TGF- $\beta 1$ and increasing doses of E2. (B) Representative western blots showing total protein levels of Smad4 in TAFs treated with TGF- $\beta 1$ alone or with TGF- $\beta 1$ and increasing doses of E2. (C) Representative western blots showing the time-dependent decrease in total protein levels of RhoA in TAFs pre-treated with TGF- $\beta 1$ and then incubated with E2. (D) Representative western blots showing total protein levels of RhoA, Rac1, ROCK1 and ROCK2 in TAFs treated with TGF- $\beta 1$ alone or with TGF- $\beta 1$ and increasing doses of E2. As regards the blots, lanes from left to right indicate the same concentrations as those indicated in the bar charts below (bars from left to right). Three independent experiments were performed. 


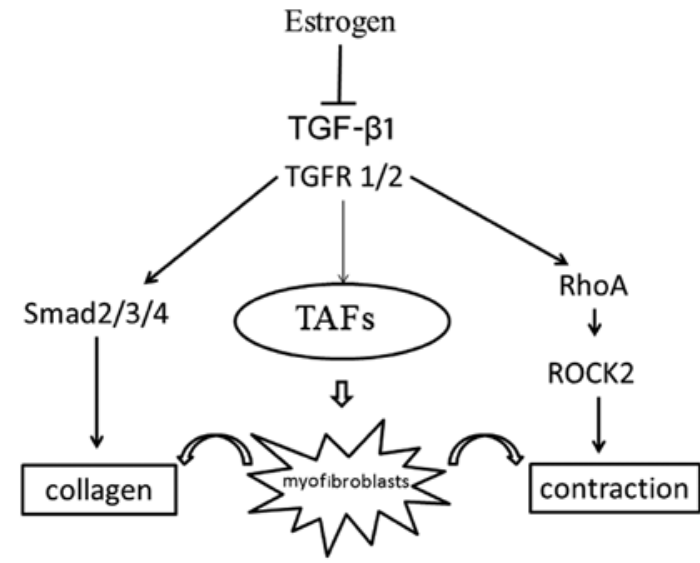

Figure 6. Proposed model of estrogen-mediated inhibition of tunica albugineaderived fibroblasts (TAFs). 17ß-estradiol (E2) suppresses the transformation of TAFs into myofibroblasts, which in turn alters the secretion of collagen and the contraction of the cells. E2 partially inhibits collagen secretion by modulating the transforming growth factor- $\beta 1$ (TGF- $\beta 1) /$ Smad signaling pathway and reduces myofibroblast contraction by modulating the RhoA/ROCK 2 pathway.

\section{Discussion}

PD is characterized by fibrosis of the TA. TGF- $\beta$ is crucial to tissue regeneration and remodeling in the TA, as has been demonstrated in previous in vivo and in vitro studies $(27,28)$. Estrogen is a type of sex hormone that is present in both men and women and is found in particularly high levels in women of reproductive age. The major functions of estrogen include the development of female secondary sexual characteristics, the thickening of the endometrium, and other aspects of menstrual cycle regulation $(29,30)$. In males, estrogen regulates certain functions of the reproductive system that play an important role in the maturation of sperm (31) and may be necessary for a healthy libido (32). Previous studies have suggested that exposure to estrogen has long-term benefits, preventing the progression of fibrotic disease (11-15). However, there is little detailed information currently available as to the mechanisms through which estrogen affects PD. TAFs have been shown to be important to the pathological process of PD. Thus, in the present study, we examined the effects of estrogen (E2) on the activation of cultured TAFs in vitro, as well as the possible mechanisms involved. Our results revealed the following: i) TGF- $\beta 1$ increased $\alpha$ SMA expression in cultured TAFs in a concentration-dependent manner, and the maximal protein expression of $\alpha$ SMA was noted in the TAFs treated with $10 \mathrm{ng} / \mathrm{ml} \mathrm{TGF}-\beta 1$ for $24 \mathrm{~h}$; ii) E2 partially inhibited the transformation of TAFs into myofibroblasts; iii) E2 attenuated the TGF- $\beta 1$-induced collagen production, as well as the contraction of myofibroblasts; iv) the phosphorylation of Smad2 was enhanced in the TAFs following stimulation with TGF- $\beta 1$ and was significantly suppressed following exposure to E2; v) TGF- $\beta 1$ stimulation increased the expression of RhoA and ROCK2, although the expression of these proteins was decreased following treatment with E2.

TAFs represent the largest class of cells that make up the normal TA, and the phenotypic transformation of TAFs into myofibroblasts is the main characteristic of PD. Under abnormal conditions, the persistence of myofibroblasts can facilitate fibrosis, which results in the structural remodeling of the penis (33). Therefore, preventing myofibroblast transformation is a potential therapeutic strategy to limit the fibrotic transformation of the TA. Myofibroblasts are highly active cells that express $\alpha \mathrm{SMA}$. In this study, we employed western blot analysis and immunofluorescence staining and found that E2 inhibited TAF activation, which was indicated by a dose-dependent decrease in $\alpha$ SMA expression (Figs. 1 and 2). Additionally, we found that activated myofibroblasts synthesized and secreted increased levels of collagen proteins and possessed contractile function similar to smooth muscle cells. We demonstrated that TGF- $\beta 1$ elevated the level of hydroxyproline (an amino acid that found only in collagen) in the TAF supernatant and that E2 suppressed this elevation (Fig. 3). The results that we obtained using cultured TAFs confirmed the beneficial effects of E2, which have also been demonstrated in previous studies $(34,35)$. We also found that TGF- $\beta 1$ significantly enhanced the contraction of primary TAFs in a collagen gel contraction assay and that the addition of E2 partly negated this effect (Fig. 4). Therefore, we suggest that the appropriate supplementation of E2 is an effective strategy for the treatment of PD.

Smad proteins are thought to play an important role in regulating intracellular responses to TGF- $\beta 1$. Following the TGF- $\beta$ 1-induced phosphorylation of Smad2 and Smad3, these proteins have been shown to localize to the nucleus and form a complex with Smad4, which mediates collagen expression (36-38). In accordance with these observations, our study demonstrated that TGF- $\beta 1$ induced Smad 2 phosphorylation in TAFs. However, we also found that E2 significantly suppressed the expression of $\mathrm{p}-\mathrm{Smad} 2$ in TAFs exposed to TGF- $\beta 1$. Additionally, the expression of Smad4 was reduced in the TAFs treated with a combination of $\mathrm{E} 2$ and TGF- $\beta 1$ compared to those treated with TGF- $\beta 1$ alone. These findings indicate that E2 blocks the TGF- $\beta 1$-induced production of $\mathrm{p}$-Smad 2 in TAFs and inhibits their secretion of collagen. We suggest a possible mechanism to explain these findings, which is that E2 attenuated TGF- $\beta 1$-induced Smad 2 phosphorylation and therefore inhibited Smad complex formation through interactions with the $\mathrm{G}$ protein-coupled estrogen receptor (GPER).

RhoA, a member of the Ras homolog gene family is a small guanosine triphosphate (GTP)ase known to regulate the actin cytoskeleton during the formation of stress fibers. Previous studies have suggested that the small GTP-binding protein Rho and its downstream targets, ROCK (including ROCK1 and ROCK2), play important roles in numerous fibrotic diseases (39). By phosphorylating the myosin light chain (MLC), Rho GTPase also induces $\mathrm{Ca}^{2+}$ sensitization during the contraction of various cells (40). It is well known that the Rho/ROCK pathway is a TGF- $\beta$-Smad independent pathway (8). In agreement with these aforementioned studies these results, we found that the RhoA/ROCK2 pathway became was activated in TAFs treated with TGF- $\beta 1$, which then enhanced myofibroblast contractility. By contrast, E2 clearly hindered the TGF- $\beta 1$-induced upregulation of RhoA and ROCK2 proteins. However, our results demonstrated that the expression levels of Rac1 and ROCK1 in TAFs were not markedly decreased when TGF- $\beta 1$ was supplemented with E2. This discovery indicates that E2 hinders the ability of TGF- $\beta 1$ to contract TAFs through the modulation of the 
RhoA-ROCK2 signaling pathway. Moreover, it has previously been revealed that the inhibition of the RhoA/ROCK pathway results in the relaxation of smooth muscle in the penis (41), thereby enhancing erectile dysfunction, which is a common symptom of PD.

In conclusion, the findings of the present study demonstrate that E2 inhibits the transformation of TAFs into myofibroblasts, reduces the expression of collagen through the modulation of the TGF- $\beta 1$-Smad signaling pathway, and suppresses the contraction of myofibroblasts through the modulation of the RhoA-ROCK2 signaling pathway (Fig. 6). These results suggest that E2 attenuates the development of PD. However, further studies are warranted to elucidate the detailed mechanisms of action of estrogen in PD in vitro and in vivo.

\section{Acknowledgements}

The present study was supported by grants from the National Natural Science Foundation of China (nos. 81170563 and 81270694).

\section{References}

1. Langston JP and Carson CC III: Peyronie's disease: review and recent advances. Maturitas 78: 341-343, 2014

2. Jarow JP and Lowe FC: Penile trauma: an etiologic factor in Peyronie's disease and erectile dysfunction. J Urol 158 1388-1390, 1997.

3. El-Sakka AI, Hassoba HM, Chui RM, Bhatnagar RS, Dahiya R and Lue TF: An animal model of Peyronie's-like condition associated with an increase of transforming growth factor beta mRNA and protein expression. J Urol 158: 2284-2290, 1997.

4. Valente EGA, Vernet D, Ferrini MG, Qian A, Rajfer J and Gonzalez-Cadavid NF: L-arginine and phosphodiesterase (PDE) inhibitors counteract fibrosis in the Peyronie's fibrotic plaque and related fibroblast cultures. Nitric Oxide 9: 229-244, 2003

5. Powell DW, Mifflin RC, Valentich JD, Crowe SE, Saada JI and West AB: Myofibroblasts. I. Paracrine cells important in health and disease. Am J Physiol 277: C1-C9, 1999.

6. Gelbard M: Myofibroblasts and mechanotransduction: do forces in the tunica albuginea contribute to Peyronie's disease? J Sex Med 5: 2974-2976, 2008.

7. Vernet D, Ferrini MG, Valente EG, Magee TR, Bou-Gharios G, Rajfer J and Gonzalez-Cadavid NF: Effect of nitric oxide on the differentiation of fibroblasts into myofibroblasts in the Peyronie's fibrotic plaque and in its rat model Nitric Oxide 7: 262-276, 2002

8. Derynck R and Zhang YE: Smad-dependent and Smadindependent pathways in TGF-beta family signalling. Nature 425: 577-584, 2003.

9. Li JH, Zhu HJ, Huang XR, Lai KN, Johnson RJ and Lan HY: Smad7 inhibits fibrotic effect of TGF-Beta on renal tubular epithelial cells by blocking Smad2 activation. J Am Soc Nephrol 13: 1464-1472, 2002.

10. Schofield AV and Bernard O: Rho-associated coiled-coil kinase (ROCK) signaling and disease. Crit Rev Biochem Mol Biol 48: 301-316, 2013.

11. Di Martino V,Lebray P, Myers RP, Pannier E, Paradis V, Charlotte F Moussalli J, Thabut D, Buffet C and Poynard T: Progression of live fibrosis in women infected with hepatitis $\mathrm{C}$ : long-term benefit of estrogen exposure. Hepatology 40: 1426-1433, 2004

12. Yang JD, Abdelmalek MF, Pang H, Guy CD, Smith AD, Dieh AM and Suzuki A: Gender and menopause impact severity of fibrosis among patients with nonalcoholic steatohepatitis Hepatology 59: 1406-1414, 2014

13. Smyk DS, Rigopoulou EI, Pares A, Billinis C, Burroughs AK Muratori L, Invernizzi P and Bogdanos DP: Sex differences associated with primary biliary cirrhosis. Clin Dev Immunol 2012 610504, 2012.

14. Pedram A, Razandi M, O'Mahony F, Lubahn D and Levin ER: Estrogen receptor-beta prevents cardiac fibrosis. Mol Endocrinol 24: 2152-2165, 2010

15. Morani A, Barros RPA, Imamov O, Hultenby K, Arner A, Warner M and Gustafsson JA: Lung dysfunction causes systemic hypoxia in estrogen receptor beta knockout $\left(\mathrm{ERbeta}^{-1 /}\right)$ mice. Proc Natl Acad Sci USA 103: 7165-7169, 2006.

16. Dixon A and Maric C: 17beta-Estradiol attenuates diabetic kidney disease by regulating extracellular matrix and transforming growth factor-beta protein expression and signaling. Am J Physiol Renal Physiol 293: F1678-F1690, 2007.
17. Novotný M Vasilenko T, Varinská L, Smetana K Jr, Szabo P Sarišský M, Dvořánková B,Mojžiš J, Bobrov N, Toporcerová S, et al: ER- $\alpha$ agonist induces conversion of fibroblasts into myofibroblasts, while ER- $\beta$ agonist increases ECM production and wound tensile strength of healing skin wounds in ovariectomised rats. Exp Dermatol 20: 703-708, 2011 .

18. Ahuja SK, Sikka SC and Hellstrom WJG: Stimulation of collagen production in an in vitro model for Peyronie's disease. Int J Impo Res 11: 207-212, 1999

19. Illsley MC, Peacock JH, McAnulty RJ and Yarnold JR: Increased collagen production in fibroblasts cultured from irradiated skin and effect of TGF beta(1)- clinical study. Br J Cancer 83: 650-654, 2000.

20. Wong $M$ and Mudera V: Feedback inhibition of high TGF-beta1 concentrations on myofibroblast induction and contraction by Dupuytren's fibroblasts. J Hand Surg Br 31: 473-483, 2006.

21. Kumar A, Ruan M, Clifton K, Syed F, Khosla S and Oursler MJ TGF- $\beta$ mediates suppression of adipogenesis by estradiol through connective tissue growth factor induction. Endocrinology 153: 254-263, 2012

22. Woessner JF Jr: The determination of hydroxyproline in tissue and protein samples containing small proportions of this amino acid. Arch Biochem Biophys 93: 440-447, 1961

23. Sun Z, Wang C, Shi C, Sun F, Xu X, Qian W, Nie S and Han X: Activated Wnt signaling induces myofibroblast differentiation of mesenchymal stem cells, contributing to pulmonary fibrosis. Int J Mol Med 33: 1097-1109, 2014

24. Lauriol J, Keith K, Jaffré F, Couvillon A, Saci A, Goonasekera SA, McCarthy JR, Kessinger CW, Wang J, Ke Q, et al: RhoA signaling in cardiomyocytes protects against stress-induced heart failure but facilitates cardiac fibrosis. Sci Signal 7: ra100, 2014.

25. Ji H, Tang H, Lin H, Mao J, Gao L, Liu J and Wu T: Rho/Rock cross-talks with transforming growth factor- $\beta /$ Smad pathway participates in lung fibroblast-myofibroblast differentiation. Biomed Rep 2: 787-792, 2014.

26. Manickam N, Patel M, Griendling KK, Gorin Y and Barnes JL: RhoA/Rho kinase mediates TGF- $\beta 1$-induced kidney myofibroblast activation through Poldip2/Nox4-derived reactive oxygen species. Am J Physiol Renal Physiol 307: F159-F171, 2014.

27. Castiglione F, Hedlund P, Van der Aa F, Bivalacqua TJ, Rigatti P Van Poppel H, Montorsi F, De Ridder D and Albersen M: Intratunical injection of human adipose tissue-derived stem cells prevents fibrosis and is associated with improved erectile function in a rat model of Peyronie's disease. Eur Urol 63: 551-560, 2013.

28. Gonzalez-Cadavid NF and Rajfer J: Experimental models of Peyronie's disease. Implications for new therapies. J Sex Med 6: 303-313, 2009.

29. McKenna TJ: Oestrogens for women: the risk/benefit ratio. Ir Med J 80: 219-221, 1987.

30. Studd J and Zamblera D: Estrogen therapy in women over 60 years of age. Gynecol Endocrinol 8: 191-196, 1994

31. Hess RA, Bunick D, Lee KH, Bahr J, Taylor JA, Korach KS and Lubahn DB: A role for oestrogens in the male reproductive system. Nature 390: 509-512, 1997.

32. Hill RA, Pompolo S, Jones MEE, Simpson ER and Boon WC: Estrogen deficiency leads to apoptosis in dopaminergic neurons in the medial preoptic area and arcuate nucleus of male mice. Mol Cell Neurosci 27: 466-476, 2004

33. Gonzalez-Cadavid NF and Rajfer J: Mechanisms of Disease: new insights into the cellular and molecular pathology of Peyronie's disease. Nat Clin Pract Urol 2: 291-297, 2005.

34. Wu M, Han M, Li J, Xu X, Li T, Que L, Ha T, Li C, Chen Q and $\mathrm{Li} Y$ : 17beta-estradiol inhibits angiotensin II-induced cardiac myofibroblast differentiation. Eur J Pharmacol 616: 155-159, 2009.

35. Imaoka M, Jindo $\mathrm{T}$ and Takasaki W: The process and development mechanism of age-related fibrosis in the pancreatic islets of Sprague-Dawley rats: Immunohistochemical detection of myofibroblasts and suppression effect by estrogen treatment. J Toxicol Pathol 26: 1-10, 2013.

36. Matsuo SE, Fiore APZP,Siguematu SM,EbinaKN,FrigugliettiCU Ferro MC, Kulcsar MA and Kimura ET: Expression of SMAD proteins, TGF-beta/activin signaling mediators, in human thyroid tissues. Arq Bras Endocrinol Metabol 54: 406-412, 2010.

37. Yuan S-M, Wang J, Hu X-N, Li D-M and Jing H: Transforming growth factor- $\beta /$ Smad signaling function in the aortopathies. Rev Bras Cir Cardiovasc 26: 393-403, 2011

38. Dong C, Li H-J, Chang S, Liao HJ, Zhang ZP, Huang $\mathrm{P}$ and Tang HH: A disintegrin and metalloprotease with thrombospondin motif 2 may contribute to cirrhosis in humans through the transforming growth factor- $\beta /$ SMAD pathway. Gut Liver 7: 213-220, 2013.

39. Thumkeo D, Watanabe $S$ and Narumiya S: Physiological roles of Rho and Rho effectors in mammals. Eur J Cell Biol 92: 303-315, 2013.

40. Fukata Y, Kimura K, Oshiro N, Saya H, Matsuura Y and Kaibuchi K: Association of the myosin-binding subunit of myosin phosphatase and moesin: dual regulation of moesin phosphorylation by Rho-associated kinase and myosin phosphatase. J Cell Biol 141: 409-418, 1998.

41. Sopko NA, Hannan JL and Bivalacqua TJ: Understanding and targeting the Rho kinase pathway in erectile dysfunction. Nat Rev Urol 11: 622-628, 2014. 